H. Meffert ${ }^{1}$

H.-P. Scherf ${ }^{2}$

H. Piazena ${ }^{3}$

\section{Systemische Wirkungen infraroter und ultravioletter Strahlen}

Ein prospektiver, plazebokontrollierter, intraindividueller Vergleich

Systemic Effects of Infrared and Ultraviolet Irradiations - A Prospective, Placebo-Controlled Intra-Individual Comparison

\section{Zusammenfassung}

Es wird über prospektive, plazebokontrollierte Untersuchungen der Wirkung von künstlich erzeugter Infrarot- (IR-), kombinierter Ultraviolett-Infrarot- (UV-IR-) bzw. Plazebo-Strahlung auf den peripheren arteriellen Blutdruck, Puls, die am Trommelfell gemessene Temperatur, Schmerzen bei entzündlich-degenerativen Erkrankungen des Bewegungsapparates, Wohlbefinden, Fitness und den 25-OH-Vitamin-D-Serumspiegel berichtet. $18 \mathrm{~Pa}-$ tienten mit schmerzhaften, entzündlich-degenerativen Erkrankungen des Bewegungsapparates, davon 13 mit arterieller Hypertonie (maximal Stadium II) wurden im intraindividuellen Vergleich bestrahlt. Die Intensität der UV-Strahlung, deren Vitamin-D-Wirksamkeit und die Intensität der IR-Strahlung entsprachen etwa derjenigen der sommerlichen Mittagssonne bei wolkenlosem Himmel im mediterranen Raum.

Die Bestrahlungen wurden gut vertragen. Dabei stieg die mittlere tympanal gemessene Temperatur um etwa $0,4^{\circ} \mathrm{C}$ (Modus UVIR) bzw. $0,6^{\circ} \mathrm{C}$ (Modus IR) an. Der mittlere arterielle Blutdruck fiel anhaltend um etwa $8 \mathrm{~mm} \mathrm{Hg}$. Schmerzen verringerten sich. Subjektiv wurden Wohlbefinden und Fitneß eindeutig gebessert. Der 25-OH-Vitamin-D-Serumspiegel stieg an.
Abstract

It is reported about prospective, placebo-controlled investigations of the effects of artificial infrared (IR), combined IR plus ultraviolet (UV) and placebo irradiations on peripheral arterial blood pressure, pulse, eardrum temperature, pain caused by inflammatory-degenerative muscle and bone disease, wellbeing, fitness and 25-OH-Vitamin D serum level. 18 patients suffering from inflammatory-degenerative muscle and bone disease, 13 out of them also of arterial hypertension (maximally stadium II) have been irradiated in an intra-individually comparing manner. All of them intensity of UV radiation, its vitamin D synthesizing capacity, and IR irradiance correspond about to the mediterranian solar irradiance at sea level during noon time in summer and at cloudless sky.

Irradiations had been tolerated well. At the same time mean temperature measured at eardrum rose approximately $0.4{ }^{\circ} \mathrm{C}$ (modus UV-IR) and $0.6^{\circ} \mathrm{C}$ (modus IR), respectively. Mean arterial blood pressure decreased continually by around $8 \mathrm{~mm} \mathrm{Hg}$. Pain, wellbeing and fitness improved clearly.

\section{Einleitung}

Die prophylaktische und therapeutische Anwendung von Solarstrahlung und Wärme ist eine weit verbreitete und auch eine der ältesten Methoden der Medizin [1]. Als nichtmedikamentö- ses Agens weist optische Strahlung (Infrarot - IR; Sichtbares VIS; Ultraviolett - UV) neben unzweifelhaften Vorzügen auch den Nachteil auf, dass die Anwendung von Verfahren der evidenzbasierten Medizin dabei meist schwierig ist. Doppelblindstudie und Plazebo-Behandlung sind Domänen der medikamen-

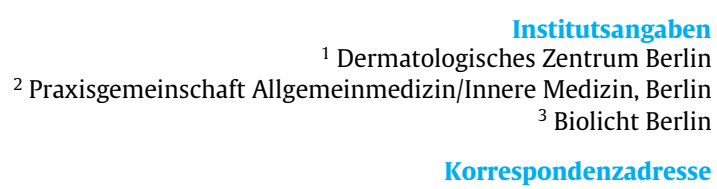

Korrespondenzadresse Prof. Dr. med. Hans Meffert • Dermatologisches Zentrum Berlin · Potsdamer Chaussee 80 • 14129 Berlin . E-mail: hans.meffert@web.de 
tösen Therapie. Zur Bewertung nichtmedikamentöser Verfahren sind einfach- oder doppelblinde Prüfungen kaum lupenrein realisierbar. Schon das einfach-blinde Bedienen leistungsfähiger Geräte ist oft weder sinnvoll noch zu verantworten. Und Plazebo-Bestrahlungen werden vom Probanden in der Regel rasch als solche erkannt.

Hier wird über kontrollierte Untersuchungen der Wirkung von künstlich erzeugter IR-, kombinierter UV-IR- bzw. PlazeboStrahlung auf den peripheren arteriellen Blutdruck, Puls, die am Trommelfell gemessene Temperatur, Schmerzen bei entzündlich-degenerativen Erkrankungen des Bewegungsapparates, Wohlbefinden und den 25-OH-Vitamin-D-Serumspiegel berichtet. Der Studie war von der Ethik-Kommission am Universitätsklinikum Charité, Berlin, zugestimmt worden (EA1/202/2005).

\section{Gestaltung der Studie}

Jeder Proband wurde mit jeder der drei Strahlenqualitäten mehrfach behandelt. Der Plazebo-Behandlung diente ein Gerät, das sich äußerlich nicht vom Verum-Gerät unterschied. Die rötliche Plazebo-Strahlung ähnelte im Farbton der im Modus IR bzw. UVIR emittierten sichtbaren Strahlung sehr. Jedoch erwärmte die Plazebo-Strahlung im Gegensatz zur IR- oder UV-IR-Strahlung die Haut nicht merklich. Deshalb wurden die Plazebo-Bestrahlungen vor den erwärmenden Modi UV-IR und IR verabfolgt. Anderenfalls hätten die Probanden das Plazebo mit großer Wahrscheinlichkeit bereits zu Beginn der Studie erkannt.

\section{Probanden}

In die Studie wurden alle diejenigen Patienten eingeschlossen, die die Allgemeinmedizinische Arztpraxis Dr. sc. med. H.-P. Scherf, Frankfurter Allee 165, 10365 Berlin, im Zeitraum vom 1. August bis 30. September 2005 wegen schmerzhafter, entzündlich-degenerativer Erkrankungen des Bewegungsapparates aufgesucht hatten und nach ausführlicher Aufklärung - auch hinsichtlich des erforderlichen zeitlichen Mehraufwands - ihre Zustimmung zu den aufwendigen diagnostischen und therapeutischen Maßnahmen gegeben hatten. Nur ein Proband mit einem Körpermasseindex (KMI) $<25$ war als normalgewichtig zu bezeichnen. Mit KMI $25<\mathrm{KMI}<30$ waren 9 Probanden übergewichtig, mit KMI > 30 waren 4 Probanden adipös. Bei einem Probanden bestand eine Hypertonie vom Borderline-Typ, bei acht Probanden bestand eine Hypertonie im Stadium I nach den WHO-Kriterien und bei vier Stadium II. Der Trainingszustand wurde aus sportärztlicher Sicht bei drei Probanden als „gut“, bei sieben als „genügend“ und bei vier als „mangelhaft“ eingestuft. Wegen anderer als der entzündlich-degenerativen Erkrankungen des Bewegungsapparates vor Studienbeginn verordnete Medikamente wurden weiter gegeben. Weitere Einzelheiten zum Patientengut sind in Tab. 1 zusammengefasst.
Tab. 1 Probanden

\begin{tabular}{|ccccc}
\hline Proband Nr. & Geschlecht & Alter (Jahre) & Größe (cm) & Gewicht $(\mathbf{k g})$ \\
\hline 1 & m & 65 & 178 & 92,0 \\
\hline 2 & m & 58 & 172 & 83,5 \\
\hline 3 & m & 67 & 180 & 80,6 \\
\hline 4 & m & 51 & 176 & 82,3 \\
\hline 5 & m & 67 & 168 & 79,0 \\
\hline 6 & m & 47 & 180 & 99,0 \\
\hline 7 & w & 51 & 184 & 121,1 \\
\hline 8 & w & 46 & 166 & 82,9 \\
\hline 9 & m & 64 & 173 & 77,4 \\
\hline 10 & w & 72 & 164 & 85,1 \\
\hline 11 & m & 43 & 176 & 84,6 \\
\hline 12 & m & 69 & 172 & 74,9 \\
\hline 13 & m & 60 & 164 & 66,3 \\
\hline 14 & w & 74 & 168 & 72,6 \\
\hline
\end{tabular}

Tab. 2 Darstellung und Bewertung von Eigenschaften eines Bestrahlungsgeräts vom Typ Innergize HB935 nach Messungen mit einem Radiometer vom Typ OL 754 Optronic

\begin{tabular}{|lcc|}
\hline Messgröße & Modus & \\
\hline & UV-IR & IR \\
\hline$E_{U V-C}\left[\mathrm{~W} \times \mathrm{m}^{-2}\right]$ & $<10^{-4}$ & $<10^{-5}$ \\
$\mathrm{E}_{\mathrm{UV}-\mathrm{B}}\left[\mathrm{W} \times \mathrm{m}^{-2}\right]$ & 1,14 & $<10^{-4}$ \\
\hline $\mathrm{E}_{\mathrm{UV}-\mathrm{A}}\left[\mathrm{W} \times \mathrm{m}^{-2}\right]$ & 51,1 & $<0,01$ \\
$\mathrm{E}_{\mathrm{VIS}}\left[\mathrm{W} \times \mathrm{m}^{-2}\right]$ & 80,8 & 31,0 \\
\hline $\mathrm{E}_{\mathrm{er}}\left[\mathrm{W} \times \mathrm{m}^{-2}\right]$ & 0,19 & \\
$\mathrm{E}_{\mathrm{D} 3}\left[\mathrm{~W} \times \mathrm{m}^{-2}\right]$ & 0,3 & \\
\hline $\mathrm{T}_{\mathrm{er}}[\mathrm{min}]$ & 21,8 & \\
\hline
\end{tabular}

Dabei bedeuten, in $65 \mathrm{~cm}$ Abstand zum UV-Strahlen-Austrittsfenster gemessenen,

- $E_{U V-C}, E_{U V-B}, E_{U V-A}$ und $E_{V I S}$ - die Bestrahlungsstärken in den Bereichen UV-C, UV-B, UV-A und VIS (Sichtbares)

- $E_{e r}$ - die erythemwirksame Bestrahlungsstärke gemäß CIE

- $E_{D 3}$ - die effektive Bestrahlungsstärke zur Stimulation der

Vitamin- $D_{3}$-Synthese gemäß DIN 5031-10

- $\mathrm{T}_{\mathrm{er}}$ - die Expositionsdauer zum Erreichen der Minimalen Erythemdosis (MED, $250 \mathrm{~J} \times \mathrm{m}^{-2}$ ) [8].

\section{Bestrahlungsgeräte}

Zwei Geräte vom Typ Innergize HB935 (Philips, Tussendiepen 2, 9206-AD, Drachten, Niederlande) wurden von der Firma Philips zur Verfügung gestellt (Tab.2). Die Geräte konnten im Modus „IR“ und im Modus „UV-IR“ betrieben werden. Gerätevermessungen erfolgten mit einem temperaturstabilisierten Doppelmonochromator-Spektralradiometer (Typ OL 754, Optronic Inc., Orlando, Fl., USA) mit einer Ulbrichtschen Kugel als Empfänger sowie mit einem thermoelektrischen Messgerät LM2 (Carl Zeiss Jena). Außerdem wurden zwei von den Verum-Geräten äußerlich nicht unterscheidbare sogenannte Plazebo-Geräte verwendet, die lediglich etwas Licht emittierten. 
Bestrahlungen

Die Probanden nahmen im Untersuchungsraum mindestens eine Stunde vor Beginn der Behandlung Platz. 20 Minuten vor Beginn der jeweiligen Bestrahlung wurden am akklimatisierten, bequem sitzenden Probanden Puls, Blutdruck und tympanale Temperatur gemessen. Zehn Minuten später folgten am liegenden Patienten in einminütigen Abständen insgesamt 10 weitere Messungen (Blutdruckentspannungstest nach Hecht [2]). Hierbei forderte eine speziell eingewiesene Krankenschwester mit sanfter Stimme zu körperlicher und psychischer Entspannung auf. Der Proband möge sich mit geschlossenen Augen auf seinen Atemrhythmus konzentrieren. Weitere Messungen erfolgten zu Beginn und Ende der dreißigminütigen Bestrahlungen und 15 Minuten danach. Es wurde die vordere Körperhälfte des auf dem Rücken liegenden Probanden bestrahlt. Der minimale Abstand zwischen UV-Strahlen-Austrittsfenster und Haut betrug $65 \mathrm{~cm}$.

Nach einer vierwöchigen Auswaschphase wurde - mit Ausnahme der Wochenenden - einmal täglich bestrahlt. Einleitend erfolgten sechs Plazebobestrahlungen. Dann wurde an jeweils drei aufeinander folgenden Tagen zunächst dreimal UV-IR- und dann dreimal IR-bestrahlt. Die IR-Bestrahlungsstärke und -dosis war im Modus „IR“ etwa doppelt so groß wie bei „UV-IR“ (siehe unter Bestrahlungsgeräte).

Die IR-Bestrahlungsstärke wurde unter unveränderten Bedingungen im Modus „UV-IR“ zu 226,2 W $\times \mathrm{m}^{-2}$ bestimmt, im Modus „IR“ zu 464,5 W× $\mathrm{m}^{-2}$. Die IR-Bestrahlungsstärke der Plazebo-Geräte war unter diesen Bedingungen nicht messbar. Bei vergleichenden Messungen mit dem thermoelektrischen Meßgerät LM2 war die IR-Bestrahlungsstärke der Verum-Geräte $>114$ fach größer als die der Plazebo-Geräte, die weniger als 4,1 W× $\mathrm{m}^{-2}$ betrug. Kurz gesagt, im Modus „IR“ wird in etwa die Wärmestrahlung der südeuropäischen Mittagssonne abgegeben, im Modus „UV-IR“ die UV-Intensität bei halber Intensität der dortigen solaren Wärmestrahlung (Tab. 2).

\section{Messung von Blutdruck, Puls und tympanaler Temperatur}

Blutdruck und Puls wurden mit einem Gerät boso-medicus memory (Bosch + Sohn GmbH \& Co, D-72417 Jungingen) gemessen. Dabei wurde eine Standardmanschette von 12 bis $13 \times 24 \mathrm{~cm}$, luftleer, $2,5 \mathrm{~cm}$ oberhalb der Ellenbeuge angebracht. Die Ellenbeuge befand sich während der Messung in Herzhöhe [4]. Die tympanale Temperatur wurde berührungslos mit einem handelsüblichen Infrarotthermometer gemessen, das auf das rechte Trommelfell gerichtet war.

\section{Bewertung von Schmerz und Wohlbefinden}

Zur Einschätzung ihres aktuellen Schmerzes wurden von den Probanden Zahlen (Scores) angegeben, wobei „0“ keinem Schmerz, „1“ gerade wahrnehmbarem Schmerz und „10“ dem denkbar schwersten Schmerz entsprach. Bewertet wurden die Regionen Rücken, Schultergürtel, Schultergelenk (rechts/links), Ellenbogen (rechts/links), Hüfte (rechts/links), Knie (rechts/ links), und Sprunggelenk (rechts/links).
Zur Einschätzung ihres aktuellen Wohlbefindens wurden von den Probanden die aus ihrer Schulzeit bekannten Schulnoten vergeben, wobei „1“ sehr gut, „2“ gut, „3“ genügend, „4“ mangelhaft und „5“ ungenügend entspricht.

\section{Gesamtbewertung durch die Probanden}

Mit Schulnoten bewerteten die Probanden eine Woche nach Abschluss der Bestrahlungsserie auch die Wirkung der unterschiedlichen Strahlenqualitäten auf Blutdruck, Schmerz, Wohlbefinden und Fitness.

\section{5-OH-Vitamin-D-Serumspiegel}

Dieser wurde mit dem Nichols Advantage ${ }^{\circledR}$ 25-Hydroxyvitamin D Assay (Nichols Institute Diagnostic, San Clemente, CA, USA) bestimmt. Der Normwert der Methode für 25-OH-Vitamin D3 wird zu 9,2-45,2 ng/ml für beide Geschlechter und alle Altersgruppen angegeben.

\section{Statistilk}

Signifikanzprüfungen erfolgten mit dem Wilcoxon-Test für verbundene Stichproben und Berechnung der linearen Regression mit dem Statistikprogramm SPSS 10.0.7 für Windows. Als „signifikant" wurden Unterschiede mit Irrtumswahrscheinlichkeiten von $\mathrm{p}<0,05^{*} ; \mathrm{p}<0,01^{* *}$ oder $\mathrm{p}<0,001^{* * *}$ bezeichnet.

\section{Ergebnisse}

\section{Tympanale Temperatur, Puls und Blutdruck}

Bei den Plazebo-Bestrahlungen änderten sich tympanale Temperatur und Blutdruck kaum (Abb.1 - 3). Die mittlere tympanal gemessene Temperatur stieg im Modus „UV-IR“ um etwa $0,4^{\circ} \mathrm{C}$ und im Modus „IR“ um etwa $0,6^{\circ} \mathrm{C}$ (Abb.1).

\section{Tympanale Temperatur bei Plazebo- $(\rightarrow-)$ UV-IR- $(\cdot \circ)$ und IR-Bestrahlung $(\rightarrow)$}

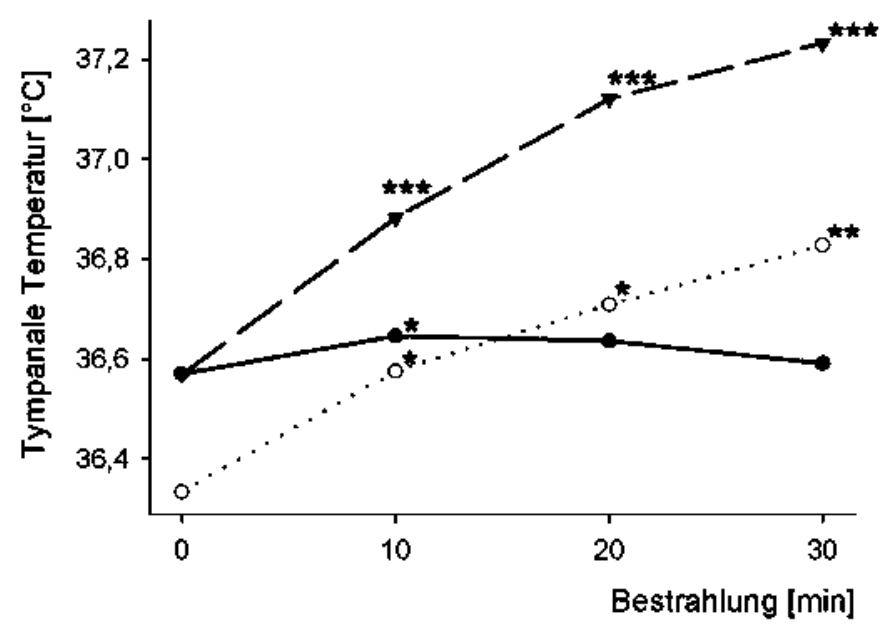

Abb. 1 Anstieg der mittleren am Trommelfell gemessenen Temperatur während der Bestrahlungen. 
Pulsfrequenz bei Plazebo- $(-\bullet)$,

UV-IR- (*0..) und IR-Bestrahlung $(\rightarrow)$

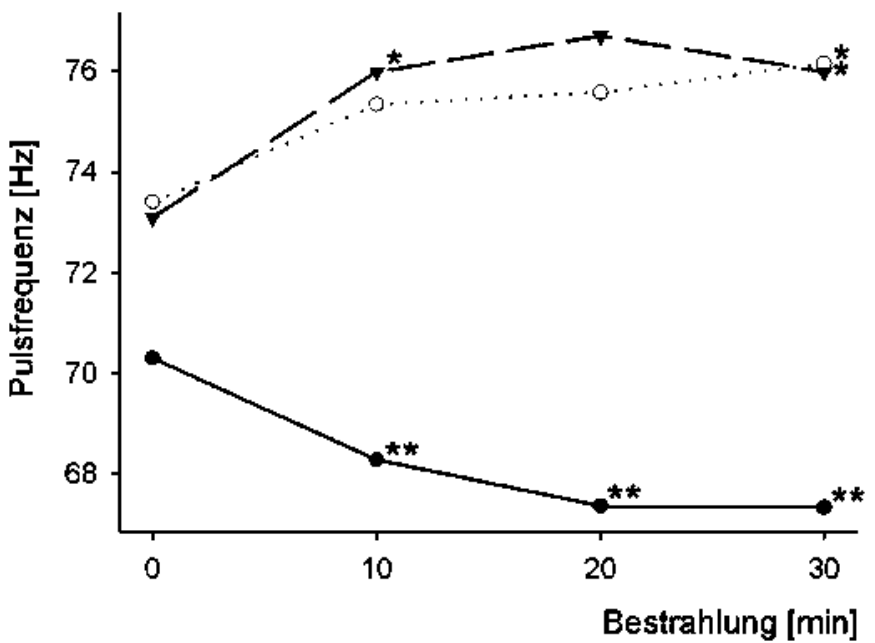

Abb. 2 Veränderungen der mittleren Pulsfrequenz während der Bestrahlungen.

\section{Systolischer Blutdruck bei Plazebo- $(\rightarrow-)$, UV-IR- (....) und IR-Bestrahlung $(\rightarrow-)$}

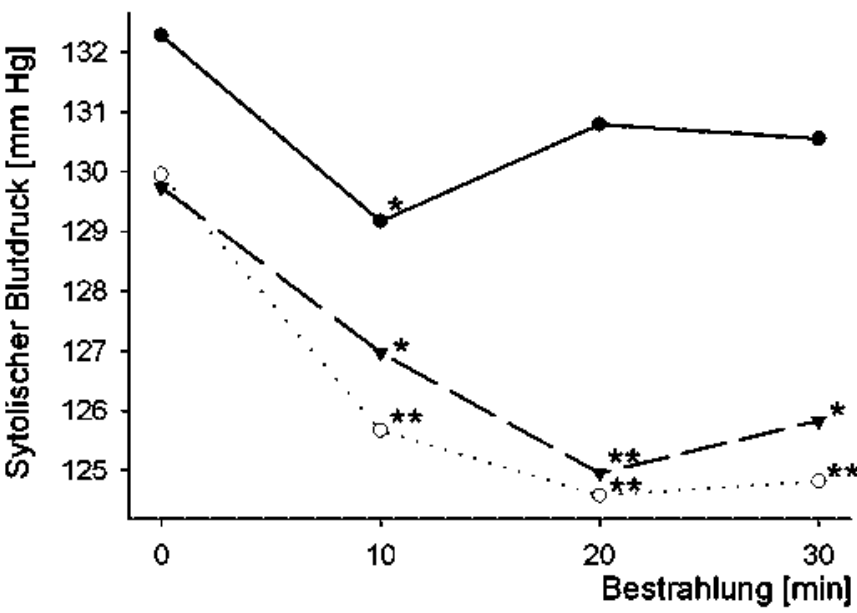

Abb. 3 Abnahme des mittleren systolischen Blutdrucks während der Bestrahlungen.

Die mittlere Pulsfrequenz fiel während der Plazebo-Bestrahlungen gering ab, während der Verum-Bestrahlungen stieg sie gering an (Abb. 2).

Der mittlere systolische Blutdruck fiel während der Plazebo-Bestrahlungen vorübergehend gering ab, während der Verum-Bestrahlungen fiel er um etwa $5 \mathrm{~mm} \mathrm{Hg}$ (Abb. 3).

Der mittlere diastolische Blutdruck änderte sich während der Plazebo-Bestrahlungen nicht, während der Verum-Bestrahlungen fiel er signifikant, wenn auch nur um wenige $\mathrm{mm} \mathrm{Hg}$ (Abb. 4).

\section{Diastolischer Blutdruck bei Plazebo- $(-\infty)$ \\ UV-IR- (-0.) und IR-Bestrahlung (- $)$}

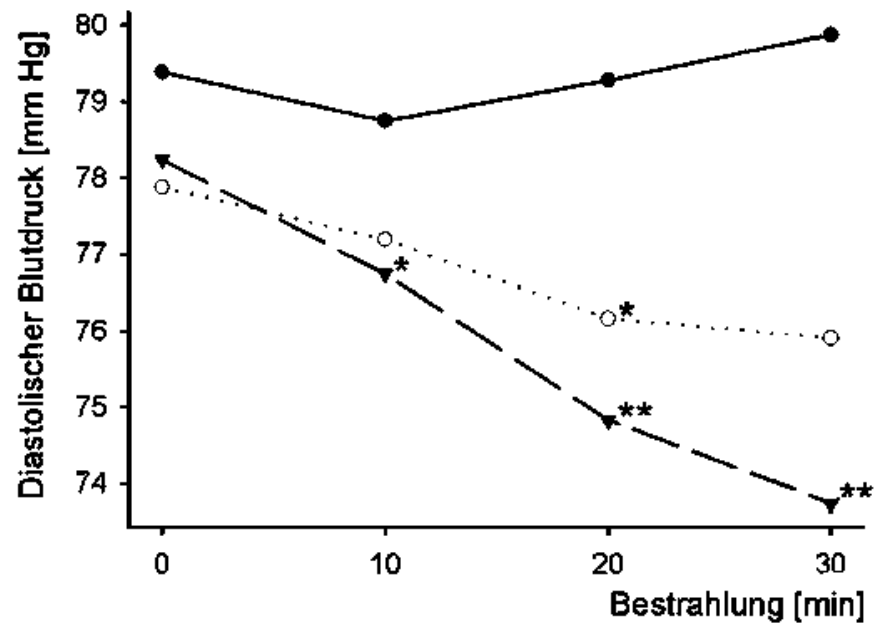

Abb. 4 Abnahme des mittleren diastolischen Blutdrucks während der Bestrahlungen.

Systolischer Blutdruck vor $(\bullet)$ und nach $(\bullet)$ den Bestrahlungen

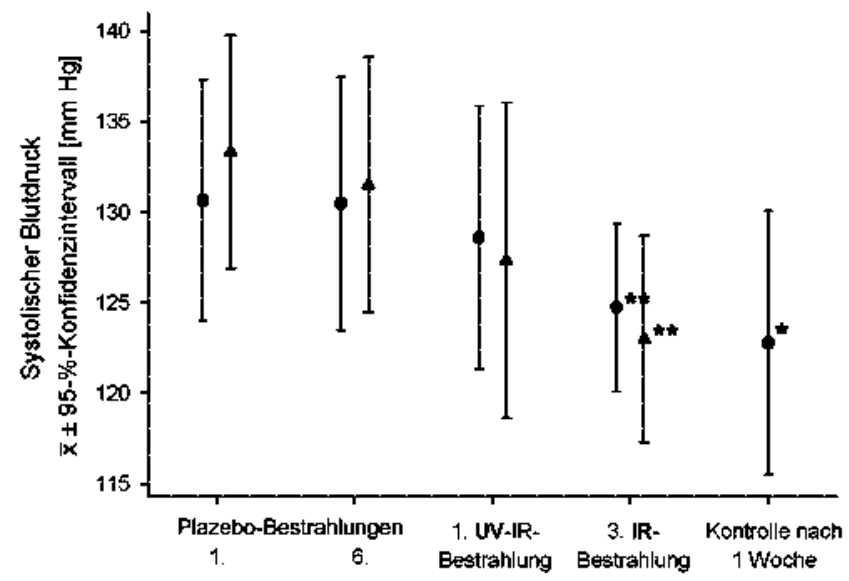

Abb. 5 Der vor der jeweiligen Bestrahlung gemessene mittlere systolische Blutdruck ist erst nach den Bestrahlungen im Modus „UV-IR“ bzw. „IR“ gesunken.

\section{Vitamin D}

Aus Abb. 7 ist ersichtlich, dass der 25-Hydroxy-Vitamin-D-Serumspiegel nach den drei UV-IR-Bestrahlungen im Oktober 2005 signifikant angestiegen war und nach einer Woche wieder auf das Ausgangsniveau sank.

\section{Schmerz und Wohlbefinden}

Die von den Betroffenen angegebene Intensität des durch entzündlich-degenerative Erkrankungen des Bewegungsapparates verursachten Schmerzes änderte sich nach den Plazebo-Bestrahlungen kaum, dagegen deutlich nach den Verum-Bestrahlungen (Abb. 8). Aufgrund des Studienablaufs kann nicht differenziert werden, welcher Modus, „IR“ oder „UV-IR“, wie viel zur mindestens eine Woche anhaltenden Schmerzlinderung beitrug. 
Sehr ähnlich fiel auch die Einschätzung der Betroffenen zum Stichwort „aktuelles Wohlbefinden“ aus (Abb.9). Nach den Plazebo-Bestrahlungen trat keine Änderung ein. Nach Bestrahlungen in den Modi „IR“ oder „UV-IR“ besserte sich das Wohlbefinden um knapp zwei Schulnoten.

\section{Gesamtbewertung durch die Probanden}

Eine Woche nach der letzten Bestrahlung wurden die Probanden nach ihrem Urteil über die Leistungsfähigkeit des Bestrahlungsgeräts hinsichtlich der Einwirkung auf Schmerz, Blutdruck, Wohlbefinden und Fitness befragt. Diese subjektive Gesamtbewertung der drei Bestrahlungsmodalitäten ergab in den Kategorien Schmerz, Blutdrucksenkung, Wohlbefinden und Fitness die Rangfolge „IR“/,UV-IR“/Plazebo (Abb.10). Nur in der Kategorie Blutdrucksenkung konnten sich die Probanden nicht entscheiden, ob „IR“ oder „UV-IR“ stärker wirkte.

\section{Diskussion}

\section{Kontrollen}

Die Untersuchungen wurden im intraindividuellen Vergleich, zeitgleich, prospektiv und plazebokontrolliert durchgeführt. Randomisierung entfiel, weil alle geeigneten Patienten in die Studie aufgenommen wurden. Dank dieses Vorgehens erkannten die Probanden die Plazebo-Bestrahlung erst im Nachhinein als solche.

Unseres Wissens wurde bislang keine derart oder besser kontrollierte Studie über die Wirkung von IR-, kombinierter UV-IR- bzW. Plazebo-Strahlung auf Parameter wie peripheren Blutdruck, Puls, tympanale Temperatur, Schmerzen bei entzündlich-degenerativen Erkrankungen des Bewegungsapparates oder Wohlbefinden publiziert.

Der periphere arterielle Blutdruck, speziell der systolische Druck, ist ein empfindlicher Indikator für physische Belastung, Stress und Entspannung. In Vorversuchen war festgestellt worden, dass der systolische Blutdruck bei bis zu 85\% der Probanden auch nach längerem Liegen (20 Minuten) auf der Bestrahlungsliege signifikant weiter abfiel. Deshalb wurde vor und nach den Bestrahlungen das Ritual des Blutdruckentspannungstests durchgeführt. An die zehn Messwerte wurde eine lineare Regressionsfunktion angepasst und deren y-Wert bei der zehnten Messung als Ausgangswert für die statistischen Berechnungen benutzt.

\section{Verträglichkeit}

Subjektiv wurden die Bestrahlungen von allen Probanden gut vertragen. Fragen nach unangenehmen Hautsensationen während der oder nach den Bestrahlungen wurden verneint. Im Modus „IR“ hatten 9 der 14 Probanden leichtes Schwitzen während der Bestrahlung angegeben. Die tympanal gemessene Temperatur reflektiert die thermische Belastung des Körperkerns. Ihr Anstieg um weniger als $1{ }^{\circ} \mathrm{C}$ in den Modi „UV-IR“ und „IR“ belegt, dass die thermische Belastung als gering einzustufen ist (Abb. 1). Die mittlere Pulsfrequenz (Abb. 2) erhöhte sich während der Bestrahlungen nur unwesentlich. Die in Tab. 3 zusammengestellten Maximalwerte der während der Bestrahlungen gemessenen Parameter Puls und tympanaler Temperatur zeigen, dass auch die Kreislaufbelastung gering ist.
Tab. 3 Während aller Bestrahlungen gemessene Maximalwerte

\begin{tabular}{|c|c|c|c|c|}
\hline $\begin{array}{l}\text { Parameter } \\
\text { Modus }\end{array}$ & $\begin{array}{l}\text { Blutdruck } \\
\text { systolisch } \\
\text { [mm Hg] }\end{array}$ & $\begin{array}{l}\text { Blutdruck } \\
\text { diastolisch } \\
\text { [mm Hg] }\end{array}$ & $\begin{array}{l}\text { Pulsfrequenz } \\
\left.\text { [min }{ }^{-1}\right]\end{array}$ & $\begin{array}{l}\text { Temperatur } \\
\text { tympanal } \\
{\left[{ }^{\circ} \mathrm{C}\right]}\end{array}$ \\
\hline \multicolumn{5}{|l|}{ Plazebo } \\
\hline Maximum & 166 & 97 & 89 & 37,6 \\
\hline Ausgangswert & 156 & 88 & 86 & 37,1 \\
\hline \multicolumn{5}{|l|}{ UV-IR } \\
\hline Maximum & 156 & 92 & 102 & 37,4 \\
\hline Ausgangswert & 141 & 92 & 86 & 36,9 \\
\hline \multicolumn{5}{|l|}{$\mathbf{I R}$} \\
\hline Maximum & 148 & 88 & 103 & 37,8 \\
\hline Ausgangswert & 158 & 92 & 92 & 37,0 \\
\hline
\end{tabular}

Diastolischer Blutdruck vor (•) und nach $(\bullet)$ den Bestrahlungen

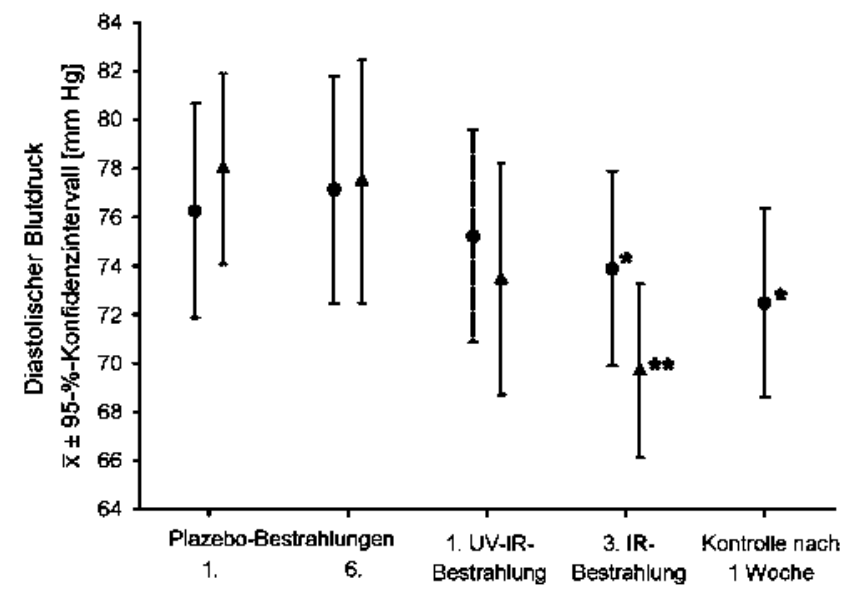

Abb. 6 Der vor der jeweiligen Bestrahlung gemessene mittlere diastolische Blutdruck ist erst nach den Bestrahlungen im Modus „UV-IR“ bzw. „IR“ gesunken.

\section{5-OH-Vitamin-D-Serumspiegel (Oktober)}

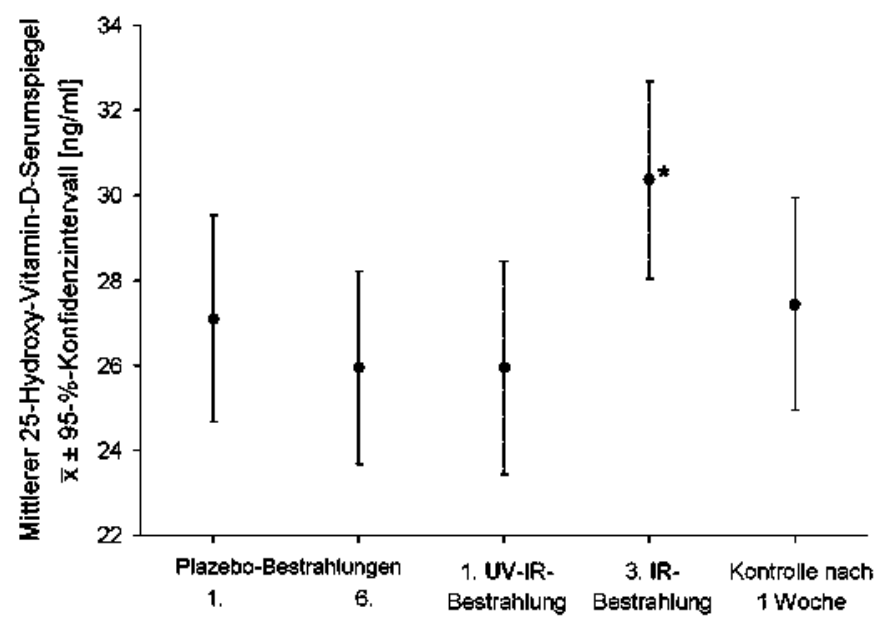

Abb. 7 Der 25-OH-Vitamin-D-Serumspiegel ist nach drei Bestrahlungen im Modus „UV-IR“ angestiegen. 
Aus Tab. 2 ist ersichtlich, dass die spektrale Verteilung der Emission des Bestrahlungsgeräts der Emission der Referenzsonne nahe kommt. Die Intensität der UV-Strahlung in $65 \mathrm{~cm}$ Abstand entspricht etwa derjenigen der sommerlichen Mittagssonne bei wolkenlosem Himmel in Mittelitalien oder Nordspanien. Gleiches gilt auch für die Vitamin-D-Wirksamkeit im Modus „UV-IR“ und die IR-Bestrahlungsstärke im Modus „IR“.

\section{Wirksamkeit serieller Bestrahlungen Blutdrucksenkung}

Bekanntlich kann schweißtreibende körperliche Aktivität den peripheren arteriellen Blutdruck anhaltend um etwa 5-10 mm Quecksilbersäule senken [4,5]. Die dabei auftretende zeitweilige Überwärmung des Körperinneren soll die Senkung des Blutdruckes auslösen. Gleichartige Wirkungen wurden mit wassergefilterter Infrarotstrahlung (IR-A, Wellenlängen von 780-1500 nm) erzielt [1].

Der jeweils vor der Bestrahlung gemessene Blutdruck gibt Auskunft über anhaltende (haftende) Effekte. Aus Abb. 5 ist ersichtlich, dass zum Zeitpunkt vor der dritten IR-Bestrahlung der mittlere arterielle Blutdruck um etwa $8 \mathrm{~mm} \mathrm{Hg}$ gefallen war. Dieser Effekt haftete über mindestens eine Woche. Gleichartig, wenn auch weniger stark ausgeprägt, verhielt sich der mittlere diastolische Blutdruck (Abb.6). Aufgrund des Studienablaufs kann nicht unterschieden werden, ob die anhaltende Blutdrucksenkung eine Folge der IR- oder der UV-IR-Bestrahlung oder beider war.

\section{Vitamin D}

Eine ausreichende Vitamin-D-Versorgung ist nicht nur für den Schutz vor Rachitis, Osteomalazie und Osteoporose erforderlich. Neuere Untersuchungen kamen zu dem Ergebnis, dass VitaminD-Mangel die Entstehung von Kolon-, Rektum- und Prostatakarzinomen, Lymphomen, entzündlichen Darmerkrankungen, Tuberkulose, Lupus erythematodes, rheumatoider Arthritis, Diabetes mellitus Typ I, multipler Sklerose, metabolischem Syndrom und Hypertonie begünstigt [6]. Der aus Abb. 8 ersichtliche geringe aber statistisch signifikante Anstieg des 25-Hydroxy-VitaminD-Serumspiegels besagt, dass durch Bestrahlungen im Modus „UV-IR“ der 25-Hydroxy-Vitamin-D-Serumspiegel erhöht werden kann. Drei Expositionen sind aber offenbar nicht ausreichend.

\section{Schmerz, Wohlbefinden und Fitness}

Nach Bestrahlungen in den Modi „IR“ und „UV-IR“ änderte sich die Intensität des durch entzündlich-degenerative Erkrankungen des Bewegungsapparates verursachten Schmerzes deutlich (Abb. 8). Die Minderung des Schmerzes hielt mindestens eine Woche an. Das ist ein von der klassischen Wärmetherapie her bekanntes Phänomen $[1,5]$. Auch die Wirkung der IR- und UV-IRBestrahlungen auf Schmerz, Blutdruck, Wohlbefinden und Fitness wurde von den Probanden günstig beurteilt (Abb. 9). Dagegen wurde nach den Plazebo-Bestrahlungen keine Änderung dieser Parameter bemerkt. Nach Bestrahlungen in den Modi „IR“ oder „UV-IR“ besserte sich das Wohlbefinden um knapp zwei Schulnoten.

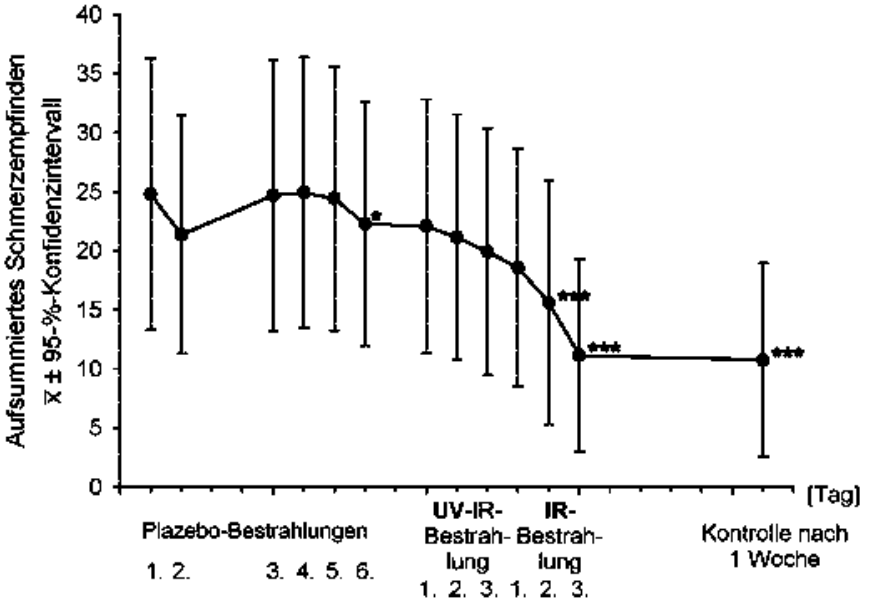

Abb. 8 Schmerzen am Bewegungsapparat sind nach sechs PlazeboBestrahlungen gering vermindert (Plazebo-Effekt). Deutliche Linderung dieser Schmerzen werden erst nach Bestrahlungen im Modus „IR“ angegeben.

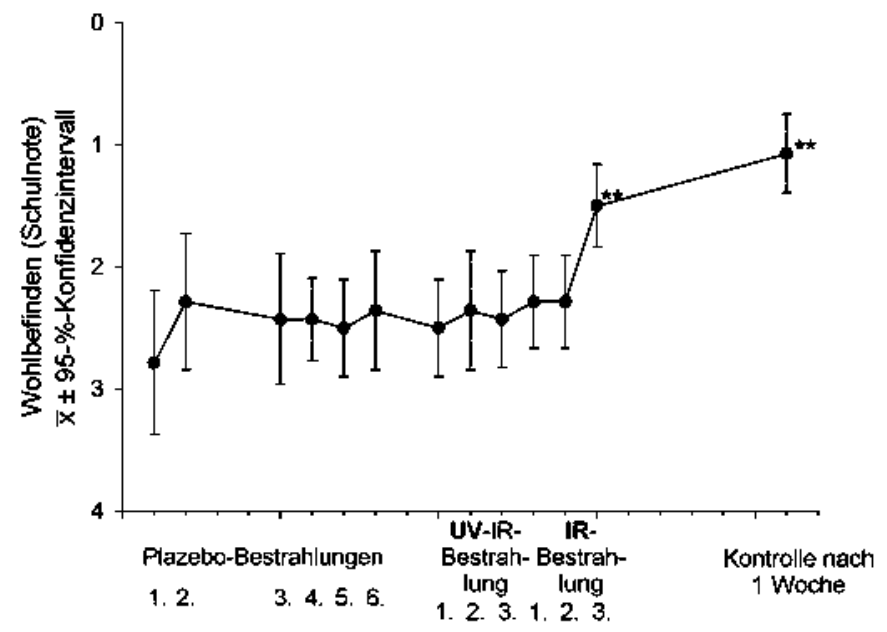

Abb. 9 Besserung des Wohlbefindens wird erst nach Bestrahlungen im Modus „IR“ angegeben.

Einschätzung der Wirkungen der Therapiemethoden

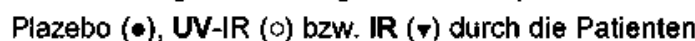

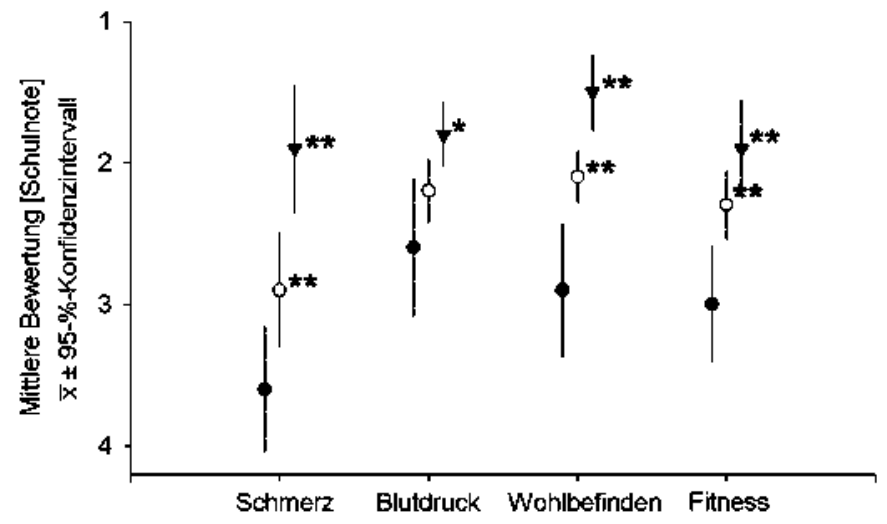

Abb. 10 Nach Abschluss der Bestrahlungsserie stellten die Probanden für die vier Kategorien die Rangfolge „IR“/,UV-IR“/Plazebo auf. 
Die vorgelegten Ergebnisse zeigen, dass serielle IR- bzw. UV-IRAnwendungen den Blutdruck anhaltend senken und durch entzündlich-degenerative Erkrankungen des Bewegungsapparates hervorgerufene Schmerzen lindern und Wohlbefinden und Fitness verbessern können. Das Verfahren empfiehlt sich für körperlich weniger belastbare Personen. Weitere Untersuchungen sollten auf die Erfassung der Langzeitwirkung gerichtet sein und Möglichkeiten der Kombination mit medikamentöser Therapie ausloten.

\section{Schlussbemerkungen}

Da UV-Strahlung ein bekanntes Karzinogen ist, sollte sie zurückhaltend angewandt werden. Es empfiehlt sich auf jeden Fall, die vom Bundesamt für Strahlenschutz bzw. „Runder Tisch Solarien“ [7] definierten aktuellen Limitierungen einzuhalten. Inwieweit größere Mengen von IR-Strahlung bzw. entsprechende Temperaturerhöhung karzinogen oder protektiv wirken können, ist umstritten [9-13].

\section{Literatur}

${ }^{1}$ Meffert $\mathrm{H}$, Piazena $\mathrm{H}$. Wirkungen künstlich erzeugter Infrarotstrahlung auf den Menschen. Akt Dermatol 2002; 28: 187-192

${ }^{2}$ Hecht K, König O, Scherf H-P. Emotioneller Stress durch Überforderung und Unterforderung. Berlin-Milow: Schibri-Verlag, 2001

${ }^{3}$ DIN 67501. Experimentelle Bewertung des Erythemschutzes von externen Sonnenschutzmitteln für die menschliche Haut. Berlin: Beuth Verlag, 1999

${ }^{4}$ Scholze J. Nichtmedikamentöse Basisbehandlung. In: Scholze J (Hrsg). Hypertonie. Berlin, Wien: Blackwell Wissenschafts-Verlag, 1999

${ }^{5}$ Mischke M. Wirkung einer einmaligen bzw. seriellen milden InfrarotA-Hyperthermie der Haut bei Patienten mit arterieller Hypertonie der WHO-Stadien I und II, Berlin: Med Diss Humboldt-Universität zu Berlin, 1990

${ }^{6}$ Peterlik M, Cross HS. Vitamin D and calcium deficits predispose for multiple chronic diseases (Review). European J Clin Invest 2005; 35 : $290-306$

7 info@bfs.de; www.bfs.de

8 Piazena $\mathrm{H}$, Meffert H. Photobiologische Bewertung von UV-TherapieGeräten. Akt Dermatol 1999; 25: 334-339

9 Schieke SM, Schroeder P, Krutmann J. Cutaneous effects of infrared radiation: from clinical observations to molecular response mechanisms (Review). Photodermatol Photoimmunol Photomed 2003; 19: 228 234

${ }^{10}$ Van der Leun JC, de Gruijl FR. Climate change and skin cancer. Photochem Photobiol Sci 2002; 1: 324-326

${ }^{11}$ Applegate LA, Scaletta C, Panizzon R et al. Induction of the putative protective protein ferritin by infrared radiation: Implications in skin repair. Internat J Molec Med 2000; 5: 247-251

12 Meneces S, Coulomb B, Lebreton C, Dubertret L. Noncoherent near infrared radiation protects normal human dermal fibroblasts from solar ultraviolet toxicity. J Invest Dermatol 1998; 111: 629-633

${ }^{13}$ Danno K, Mori N, Toda K et al. Near-infrared radiation stimulates cutaneous wound repair: laboratory experiments on possible mechanisms. Photodermatol Photoimmunol Photomed 2001; 17: 261 -265

\section{Buchbesprechung}

\section{Antike Medizin - Ein Lexikon}

\section{K.-H. Leven}

München: C. H. Beck, 2005. 967 S. Geb. 49,90€.

ISBN 3-406-52891-0

Die antike Medizin, die Anfänge also der Medizingeschichte, ist Teil der antiken Kulturgeschichte. Ursprünge, Erfolge und Fehleinschätzungen lassen sich dort schon auffinden und sie sind oft über viele Jahrhunderte zu verfolgen. Ein Lexikon der antiken Medizin ist deshalb wertvoll, hilfreich und zu begrüßen. Nun lieg es vor und imponiert.

90 Experten aus der alten und der neuen Welt haben mitgewirkt, über 1000 Stichworte bearbeitet und in gedrängter Form dargestellt. Knappe Literaturangaben belegen die einzelnen Hinweise und geben Quellen zur Vertiefung an. Ausführlich sind Literaturangaben in einem eigenen Abschnitt zusammengeführt. Ein Verzeichnis der Stichworte (Lemmata) erleichtert den Zugang.

Der Schwerpunkt ist auf die griechische und römische Antike gelegt, nicht auf die Frühphasen in Ägypten und Mesopotamien. Personen und Götter sind erfasst sowie Krankheiten, Begriffe und Behandlungen.

Und für uns Dermatologen gibt es reichlich Bezüge. Die „Hautkrankheiten“ sind erfasst und einige davon, teils Symptome, mit der Unschärfe damaliger Nomenklatur belegt. Als Referenz allerdings wird auf das Jadassohn'sche Handbuch von 1928 zurückgegriffen. Haar und Glatze, Geschlecht und Geschlechtskrankheiten sind ebenso enthalten wie auch Lepra, Anthrax, Pocken und Virus. Auch Insekten, Parasiten und Läuse kommen vor, zudem Allergie, Asthma, Juckreiz. Krebs, Geschwür, Abszess, Krampfader und Hämorrhoiden. Zu den Heilmitteln gehören viele Pflanzen und auch Thermen, Bäder, Verbände, Massage und Schröpfen. Sogar Tierversuche werden erwähnt.

Ein gelungenes und hilfreiches Lexikon.

E. G. Jung, Heidelberg 